\title{
MONOTONE METHOD FOR FIRST ORDER SINGULAR SYSTEMS WITH BOUNDARY CONDITIONS *
}

\author{
J.A. Uvah and A.S. Vatsala \\ Department of Mathematics \\ University of Southwestern Louisiana \\ P.O. Box 41010, USL \\ Lafayette, Louisiana 70504-1010
}

\begin{abstract}
Existence of maximal and minimal solutions are proved for the first order singular systems with boundary conditions by combining the method of upper and lower solutions and the monotone iterative technique.
\end{abstract}

Key words: Singular Systems, Extremal Solutions, Upper and Lower Solutions, Iterative Technique.

AMS Subject Classification: 34A34, 34B99

\section{INTRODUCTION}

It is well known [4] that by combining the method of upper and lower solutions with monotone iterative techniques, one can prove the existence of extremal solutions on nonlinear problems in a closed set, namely, the sector defined by means of the upper and lower solutions.

Recently the result has been extended [6] to singular systems with initial conditions since singular systems do occur in many physical applications. In this paper, we extend this result to singular systems with boundary conditions. This is achieved by developing the necessary comparison result. The crucial part is the consistency condition. An example is given to illustrate that such a condition is attainable.

\section{PRELIMINARY RESULTS}

Consider the boundary value problem

$$
A \dot{x}=f(t, x), \quad \operatorname{Ex}(0)=a, \quad F x(T)=b
$$

where $A \in \mathbb{R}^{n \times n}$ is singular matrix, $E, F$ are real $n \times n$ nonsingular matrices, and $f \in C^{1}\left[J \times \mathbb{R}^{n}, \mathbb{R}^{n}\right], J=[0, T]$. In this paper we combine the method of upper and lower solutions together with monotone iterative technique to prove the existence of

* Received: March, 1989; Revised: October, 1989 
extremal solutions of (1.1). For this purpose we need the existence and uniqueness of the solution of the corresponding linear boundary value problem of the form

$$
A \dot{x}+M x=g(t), \quad E x(0)=a, \quad F x(T)=b
$$

where $M^{-}$is an $n \times n$ matrix and $g$ is $n$-times differentiable on $J$. The results relative to (1.2) are well known [2,3].

Here, we recall the results without proof. Here and throughout the paper, we assume that $A$ is a singular matrix and that there exists a $\lambda \in \mathbb{R}$ for which $(\lambda A+M)^{-1}$ exists. Also, $B^{D}$ shall denote the Drazin inverse of the matrix $B$, and $\mathscr{B}$ denotes the quantity $(\lambda A+M)^{-1} B$. $(\mathscr{A}, \mathscr{M}$, and $g$ will have the same relationship to $A, M$, and $g$ )

Theorem 1.1. For a given $a, b, g$ the boundary value problem (1.2) is consistent if and only if the equation

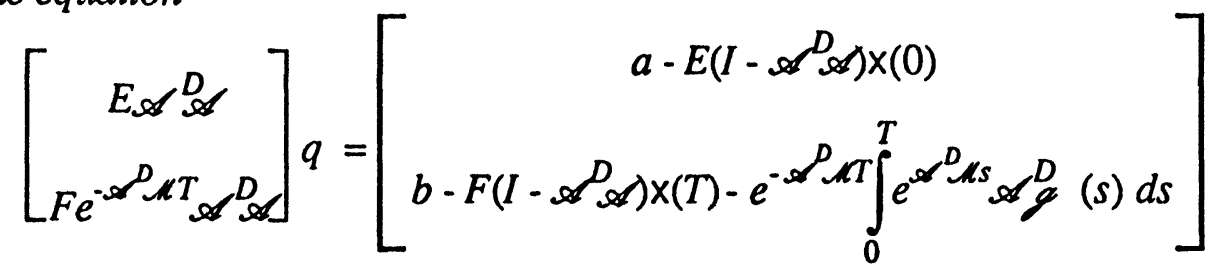

has a solution $q$.

Theorem 1.2. The boundary value problem (1.2), when consistent, has a unique solution provided that:

(a) the associated homogeneous problem

$$
A \dot{x}+M x=0, \quad E x(0)=0=F x(T)
$$

has only the zero solution; or

(b) $\operatorname{rank}(Q)=\operatorname{rank}\left(\mathscr{A}^{D} \mathscr{A}\right)=\operatorname{rank}\left(\mathscr{A}^{n}\right)$,

where 


$$
Q=\left[\begin{array}{c}
E \mathscr{A}_{\mathscr{A}}^{D} \\
F e^{-\mathscr{A}^{D} M T_{\mathscr{A}}{ }^{D}}
\end{array}\right] .
$$

One can easily show [2] that the solution of (1.2) is given by

$$
\begin{aligned}
& x(t)=e^{-\mathscr{D} \mathscr{D}} \mathscr{A}^{D} \mathscr{A} q+e^{-\mathscr{A} \mathscr{C}^{D}} \int_{0}^{t} e^{\mathscr{D} \mathscr{A}} \mathscr{A}_{g}^{D}(s) d s
\end{aligned}
$$

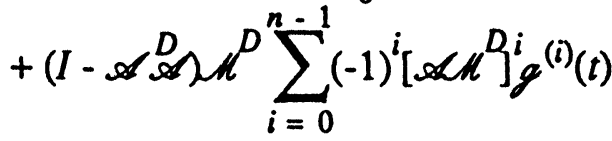

where $q$ is any vector satisfying the consistency condition.

Note 1.1: If the index of $A$ is one, the third term of (1.4) simplifies to ( $I$ $\mathscr{A} \mathscr{A} \mathscr{H}^{D} g(t)$. In this case, differentiability of $g$ is enough to show that (1.4) is in fact the solution of (1.2). Also, if the index of $A$ is one and further, if $(\lambda A+M)^{-1}$ exists, assumption ( $a$ ) of Theorem 1.2 is easily satisfied.

Next, we prove a comparison result which is needed in our main result. For convenience we list the following assumptions:

(A1) There exist $v_{0}(t), w_{0}(t) \in C^{1}\left[J, \mathbb{R}^{n}\right]$ with $v_{0}(t) \leq w_{0}(t)$ for $t \in J$ such that

$$
A \dot{v}_{0} \leq f\left(t, v_{0}\right), \quad E v_{0}(0) \leq a, \quad F v_{0}(T) \leq b
$$

$$
A \dot{w}_{0} \geq f\left(t, w_{0}\right), \quad E w_{0}(0) \geq a, \quad F w_{0}(T) \geq b
$$

That is, $v_{0}, w_{0}$ are lower and upper solutions of the boundary value problem (1.1).

(A2) There exists a matrix $M \in \mathbb{R}^{n \times n}$ such that

$$
f(t, y)-f(t, x) \geq-M(y-x)
$$

whenever 


$$
v_{0} \leq x \leq y \leq w_{0}
$$

(A3) Let $A$ and $M$ be $n \times n$ matrices such that $(\lambda A+M)^{-1}$ exists and is nonnegative for some $\lambda \in \mathbb{R}$. Also, let there exist nonnegative matrices such that

$$
P^{-1} \mathscr{A P}=\left[\begin{array}{ll}
C & 0 \\
0 & 0
\end{array}\right]
$$

where $C$ is a diagonal square matrix with $C_{j j}^{-1}>\lambda$ if $\lambda>0$, and $C_{j j}^{-1}>0$ if $\lambda<0$.

Note that for any square matrix $A$, there exist nonsingular matrices $P$ and $C$, and a nilpotent matrix $N$ such that

$$
A=P\left[\begin{array}{ll}
C & 0 \\
0 & N
\end{array}\right] P^{-1}
$$

where index of $A=$ index of $N$. Thus $N \equiv 0 \Leftrightarrow$ index of $A=1$. This in turn implies that the index of $\mathscr{A}=1$. In such a case the Drazin inverse of $\mathscr{A}$ is called the group inverse and is denoted by $\mathscr{A}^{\#}$. See [5] for details.

Theorem 1.3: Let $A \dot{p}+M p \leq 0$ such that $A$ and $M$ satisfy assumption (A3). Then $E p(0) \leq 0$ and $F p(T) \leq 0$, where $E^{-1}, F^{-1} \geq 0$, implies $p(t) \leq 0$ on $J=[0, T]$.

Proof: Set $p=P z$. Then the inequality $A \dot{p}+M p \leq 0$ reduces to $A P \dot{z}+M P z \leq 0$. Premultiplying this by $P^{-1}(\lambda A+M)^{-1}$, we have

$$
P^{-1} \mathscr{A} P \dot{z}+P^{-1} \mathscr{M} P z \leq 0
$$

since $P^{-1}$ and $(\lambda A+M)^{-1}$ are nonnegative. That is

$$
\left[\begin{array}{ll}
C & 0 \\
0 & 0
\end{array}\right]\left[\begin{array}{l}
\dot{z}_{1} \\
\dot{z}_{2}
\end{array}\right]+\left[\begin{array}{cc}
I-\lambda C & 0 \\
0 & I
\end{array}\right]\left[\begin{array}{l}
z_{1} \\
z_{2}
\end{array}\right] \leq 0
$$

This leads to $C \dot{z}_{1}+(I-\lambda C) z_{1} \leq 0$ and $z_{2} \leq 0$. Also $E p(0)=E P z(0) \leq 0$ implies $z(0) \leq 0$ since 
$P^{-1}, E^{-1}$ are nonnegative. Similarly, $z(T) \leq 0$. One can easily prove $[1,4]$ that $z_{1}(t) \leq 0$. Hence $z(t) \leq 0$ on $J$. Consequently, $P z(t) \leq 0$ or $p(t) \leq 0$ on $J$.

\section{MAIN RESULT}

Here we develop the monotone iterative technique for the singular system of the boundary value problem which yields monotone sequences that converge to the extremal solutions of $(1.1)$ relative to the sector $\left[v_{0}, w_{0}\right]$.

Theorem 2.1. Let assumptions (A1), (A2), (A3) hold such that E,F possess nonnegative inverses. Further, let the equation

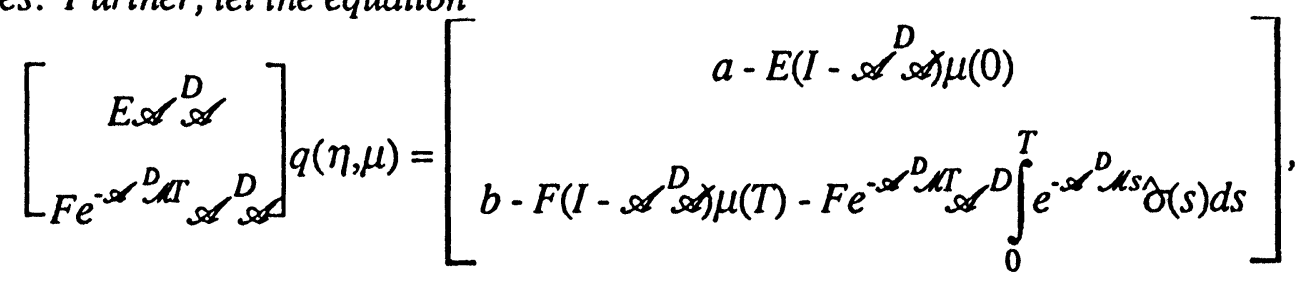

where $f(t)=(\lambda A+M)^{-1}[f(t, \eta)+M \eta]$, have a solution $q$ for each pair $\eta, \mu$ such that $v_{0}(t) \leq \eta(t) \leq w_{0}(t), v_{0}(0) \leq \mu(0) \leq w_{0}(0)$ and $v_{0}(T) \leq \mu(T) \leq w_{0}(T)$. Then there exist sequences $\left\{v_{n}\right\},\left\{w_{n}\right\}$ which converge uniformly and monotonically on $[0, T]$ to $\rho(t)$ and $\gamma(t)$, where $\rho$ and $\gamma$ are minimal and maximal solutions of (1.1) respectively. That is, if $x(t)$ is any solution of (1.1) such that $v_{0}(t) \leq x(t) \leq w_{0}(t)$ on $J$, then $\rho(t) \leq x(t) \leq \chi(t)$ on $J$.

Proof: Consider the linear boundary value problem

$$
A \dot{x}=f(t, \eta(t))-M(x-\eta(t)), \quad E x(0)=a, \quad F x(T)=b
$$

where $\eta$ belongs to the sector

$$
\left[v_{0}, w_{0}\right] \equiv\left\{u \in C^{1}\left[J, \mathbb{R}^{n}\right]: v_{0} \leq u \leq w_{0}\right\}
$$

Now (2.2) can be rewritten as

$$
A \dot{x}+M x=f(t, \eta)+M \eta, \quad E x(0)=a, F x(T)=b .
$$


Choosing $x(0)=\mu(0), x(T)=\mu(T)$, the boundary value problem (2.3) satisfies the consistency condition (2.1) and has a unique solution since the associated homogeneous problem has only the zero solution. It is easy to see this using (A3).

Define a mapping $R$ such that

$$
R \eta=x
$$

where $x$ is the unique solution of (2.3). This mapping defines the sequences $\left\{v_{n}\right\},\left\{w_{n}\right\}$. First we prove that

(a) $v_{0} \leq R v_{0}, w_{0} \geq R w_{0}$

(b) $R$ is monotone nondecreasing on the sector $\left[v_{0}, w_{0}\right]$.

To prove (a), set $R v_{0}=v_{1}$, where $v_{1}$ is the unique solution of (2.3) with $\eta=v_{0}$. Setting $p=v_{0}-v_{1}$, one can see that

$$
A \dot{p}=A \dot{v}_{0}-A \dot{v}_{1} \leq f\left(t, v_{0}\right)-f\left(t, v_{0}\right)+M\left(v_{1}-v_{0}\right)=-M p
$$

and $p(0)=v_{0}(0)-v_{1}(0) \leq 0, p(T) \leq 0$ which by using Theorem 1.3 implies $p(t) \leq 0$. That is, $v_{0}(t) \leq v_{1}(t)$ on $J$. Similarly, one can prove that $w_{0} \geq R w_{0}$ on $J$.

In order to prove (b), consider $\eta_{1}, \eta_{2}$ belonging to the sector $\left[v_{0}, w_{0}\right]$ such that $\eta_{1}(t) \leq \eta_{2}(t)$ on $J$. Set $x_{1}=R \eta_{1}, x_{2}=R \eta_{2}$ and $p(t)=x_{1}(t)-x_{2}(t)$. Then

$$
\begin{aligned}
A \dot{p}+M p & =A \dot{x}_{1}-A \dot{x}_{2}+M x_{1}-M x_{2} \\
& =f\left(t, \eta_{1}\right)+M \eta_{1}-f\left(t, \eta_{2}\right)-M \eta_{2} \\
& \leq 0
\end{aligned}
$$

using (A2). Also, $p(0)=0=p(T)$. By Theorem 1.3 this implies $p(t) \leq 0$ on $J$. That is, $x_{1}(t) \leq x_{2}(t)$ on $J$.

Now define the sequences $\left\{v_{n}\right\},\left\{w_{n}\right\}$ by

$$
v_{n+1}=R v_{n}, w_{n+1}=R w_{n} \text { for } n=0,1,2, \ldots .
$$

Using standard arguments it is easy to prove that $\lim _{n \rightarrow \infty} v_{n}=\rho(t)$ and $\lim _{n \rightarrow \infty} w_{n}=\gamma(t)$ uniformly and monotonically on $J$. It is also easy to show that $\rho(t)$ and $\gamma(t)$ are solutions of (1.1) in view of the fact that $v_{n}, w_{n}$ satisfy

$$
\begin{aligned}
& A \dot{v}_{n}=-M v_{n}+f\left(t, v_{n-1}\right)+M v_{n-1}, \quad E v_{n}(0)=a, F v_{n}(T)=b \\
& A \dot{w}_{n}=-M w_{n}+f\left(t, w_{n-1}\right)+M w_{n-1}, E w_{n}(0)=a, F w_{n}(T)=b .
\end{aligned}
$$

To prove that $\rho, \gamma$ are the minimal and maximal solutions of (1.1), it is enough to show that if $x(t)$ is any solution of (1.1) such that $v_{0} \leq x \leq w_{0}$ on $J$, then $v_{0} \leq \rho \leq x \leq \gamma \leq w_{0}$ on $J$. To do this, suppose that for some $n, v_{n} \leq x \leq w_{n}$ on $J$. Set $p=x-v_{n+1}$ so that

$$
\begin{aligned}
A \dot{p} & =A \dot{x}-A \dot{v}_{n+1} \\
& =f(t, x)-\left[-M v_{n+1}+f\left(t, v_{n}\right)+M v_{n}\right] \\
& \geq-M\left(x-v_{n}\right)+M v_{n+1}-M v_{n} \\
& =-M\left(x-v_{n+1}\right)=-M p
\end{aligned}
$$


and $p(0)=x(0)-v_{n+1}(0)=0, p(T)=x(T)-v_{n+1}(T)=0$. Using Theorem 1.3, $p(t) \geq 0$ or $x(t) \geq v_{n+1}(t)$ on $J$. Similarly, $x(t) \leq w_{n+1}(t)$ on $J$ and hence $v_{n+1} \leq x \leq w_{n+1}$ on $J$. Since $v_{0} \leq x \leq w_{0}$ on $J$, this proves by induction that $v_{n} \leq x \leq w_{n}$ on $J$ for all $n$. Taking the limit as $n \rightarrow \infty$, the theorem follows.

Below is an example to illustrate that the condition (2.1) of Theorem 2.1 is attainable.

Example 2.1: Consider the nonlinear problem

$$
A \dot{x}=f(t, x), x(0)=\left[\begin{array}{l}
0 \\
0
\end{array}\right], x(1)=\left[\begin{array}{c}
e^{-2} \\
e^{-6}
\end{array}\right],
$$

where

$$
A=\left[\begin{array}{ll}
1 & 0 \\
0 & 0
\end{array}\right], f(t, x)=\left[\begin{array}{c}
e^{-2 t}-2 x_{1} \\
x_{1}^{3}-x_{2}
\end{array}\right] .
$$

For this $f$ it is easy to see that $M$ can be chosen as $\left[\begin{array}{ll}2 & 0 \\ 0 & 1\end{array}\right]$ so that assumption (A2) is satisfied. The corresponding linear problem (2.3) is

$$
\begin{gathered}
{\left[\begin{array}{ll}
1 & 1 \\
0 & 0
\end{array}\right]\left[\begin{array}{l}
\dot{x}_{1} \\
\dot{x}_{2}
\end{array}\right]+\left[\begin{array}{ll}
2 & 0 \\
0 & 1
\end{array}\right]\left[\begin{array}{l}
x_{1} \\
x_{2}
\end{array}\right]=\left[\begin{array}{c}
e^{-2 t} \\
\eta_{1}^{3}
\end{array}\right],} \\
x(0)=\left[\begin{array}{l}
0 \\
0
\end{array}\right], x(1)=\left[\begin{array}{l}
e^{-2} \\
e^{-6}
\end{array}\right] .
\end{gathered}
$$

By direct computation, condition (2.1) reduces to the system

$$
\left[\begin{array}{cc}
1 & 0 \\
0 & 0 \\
e^{-2} & 0 \\
0 & 0
\end{array}\right]\left(\begin{array}{l}
q_{1}(\eta, \mu) \\
q_{2}(\eta, \mu)
\end{array}\right)=\left[\begin{array}{c}
0 \\
-\mu_{2}(0) \\
0 \\
e^{-6}-\mu_{2}(1)
\end{array}\right]
$$

with solution $q_{1}=0$ and $q_{2}$ arbitrary.

Remark 2.1: In the case where $a=0=b$, and $T=1$, the solution of the linear problem (1.2) can be written in the form (see [2] for details)

$$
x(t)=\int_{0}^{1} G(t, s)_{g}(\mathrm{~s}) d s+\int_{0}^{1} H(t, s)_{g}(s) d s
$$

where the matrix $G$ is given by 


$$
G(t, s)=\left\{\begin{array}{l}
Y(t) R^{+} F Y(1) Y^{-1}(s) \mathscr{A}^{D}, s<t \\
-Y(t) R^{+} F Y(1) Y^{-1}(s) \mathscr{A}^{D}, s>t
\end{array}\right.
$$

and

$$
\begin{gathered}
H(t, s)=\left(I-\mathscr{A}^{D} \mathscr{A}\right) \mathscr{A}^{D} \delta(s-t)+Y(t) R^{+} E\left(I-\mathscr{A}^{\mathrm{D}} A\right) \mathscr{M}^{D} \delta(s) \\
+Y(t) R^{+} F\left(I-\mathscr{A}^{\mathrm{D}} \mathscr{A}\right) \mathscr{N}^{D} \delta(s-1),
\end{gathered}
$$

where $R=E \mathscr{A}^{D} \mathscr{A}+F Y(1) \mathscr{A}^{D} \mathscr{A}, \delta(t)$ is the delta function, $R^{+}$denotes the generalized inverse of $R$ and $y=e^{-\infty A R t}$ is the fundamental matrix for the homogeneous problem:

$$
A \dot{x}+M x=0, E x(0)=0=F x(1) .
$$

It is to be noted in this special case that it suffices if $f \in C\left[J \times \mathbb{R}^{n}, \mathbb{R}^{n}\right]$. Now (2.4) can be used to develop the monotone method and show the existence of extremal solutions of (1.1) when $a=b=0$.

\section{REFERENCES}

[1] Bernfeld, S.R., and V. Lakshmikantham, An Introduction to Non-linear Boundary Value Problems, Academic Press, Inc. New York (1974).

[2] Campbell, S.L., Singular Systems of Differential Equations, Pitman Publishing Co., London (1980).

[3] Campbell, S.L., and C.J. Meyer, Generalized Inverses of Linear Transformations, Pitman Publishing Co., San Francisco (1979).

[4] Ladde, G.S, V. Lakshmikantham, and A. S. Vatsala, Monotone Iterative Techniques for Nonlinear Differential Equations, Pitman Publishing Co., Boston (1985).

[5] Lancaster, P., and M. Tismenetsky, The Theory of Matrices, Academic Press, New York (1985).

[6] Vatsala, A.S., Monotone Iterative Technique for Singular Systems of Differential Equations, Proc. Inter. Conf. on Nonlinear Analysis and Applications, Marcel Dekker (1987) pp. 579 - 582. 


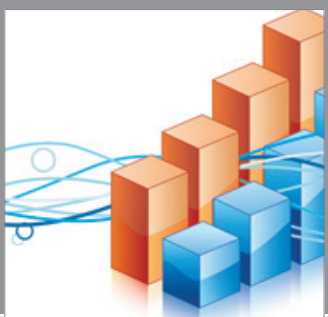

Advances in

Operations Research

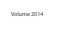

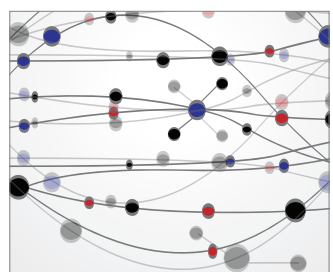

\section{The Scientific} World Journal
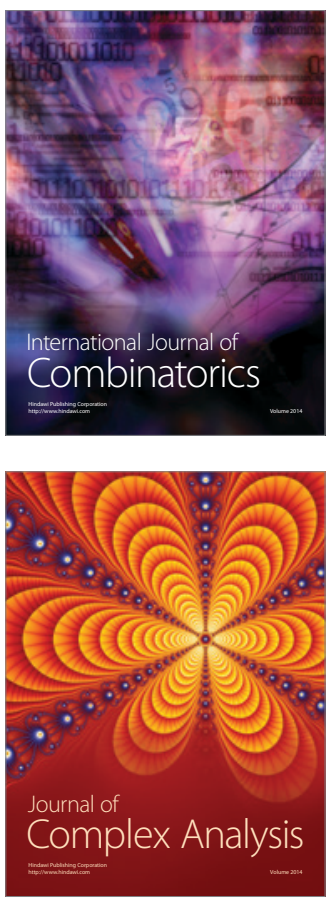

International Journal of

Mathematics and

Mathematical

Sciences
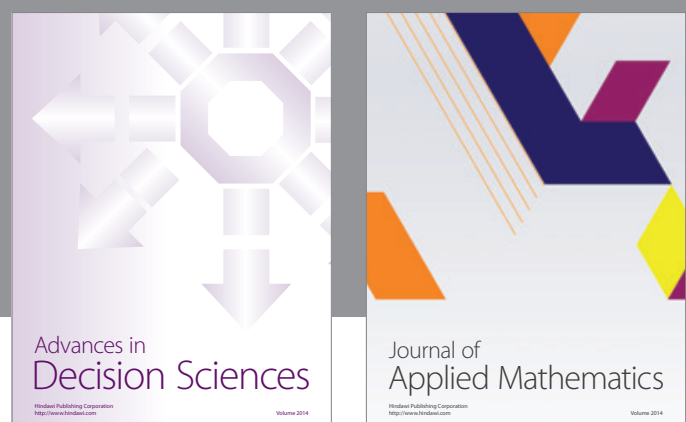

Journal of

Applied Mathematics
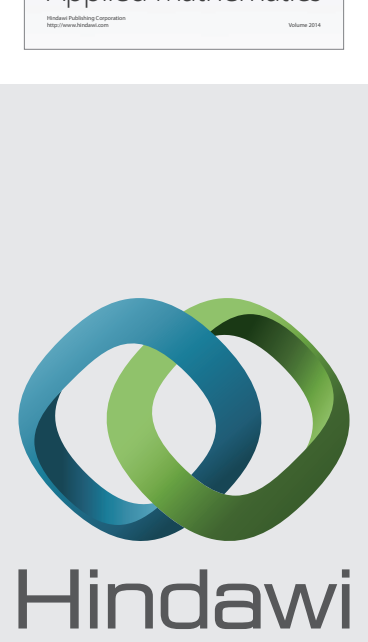

Submit your manuscripts at http://www.hindawi.com
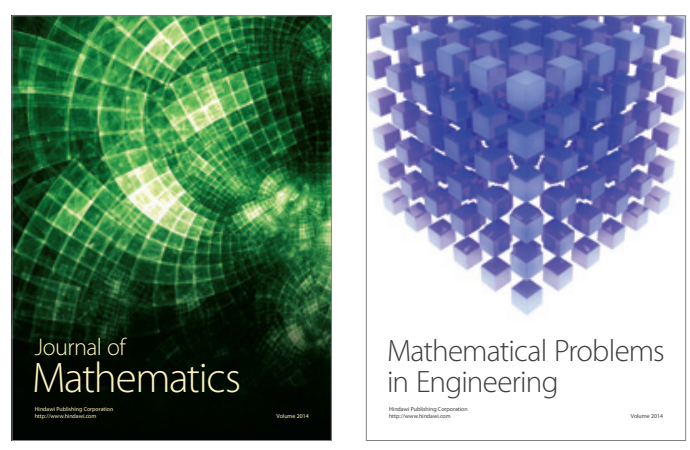

Mathematical Problems in Engineering
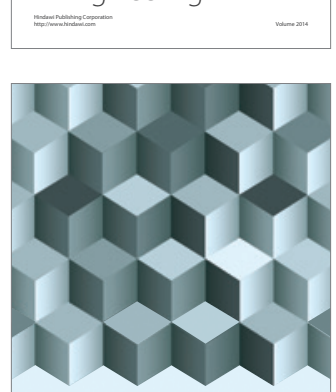

Journal of

Function Spaces
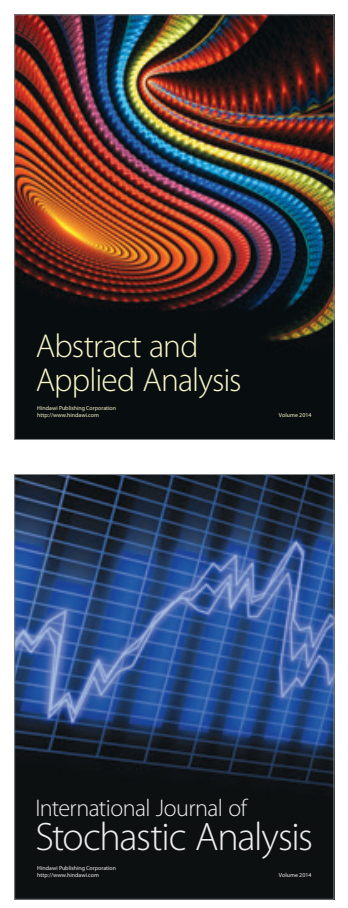

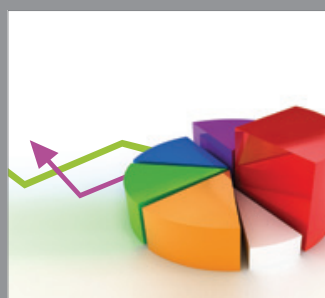

ournal of

Probability and Statistics

Promensencen
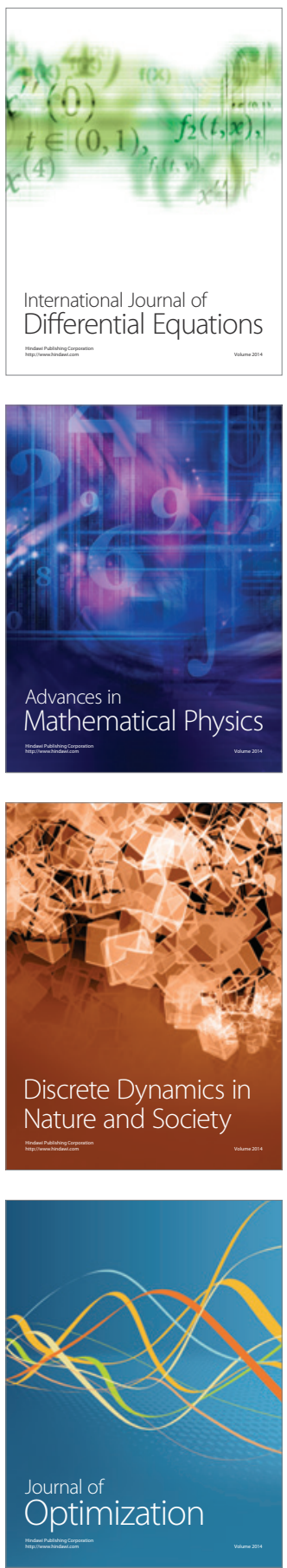